Research Article

\title{
Energy Conservation for 3D Tropical Climate Model in Periodic Domains
}

\author{
Hui Zhang \\ College of Mathematical and Physical Science, Key Laboratory of Complex Systems of Anhui Province, Anqing Normal University, \\ Anqing, 246133, China \\ Correspondence should be addressed to Hui Zhang; zhangaqtc@126.com
}

Received 10 March 2021; Accepted 3 August 2021; Published 9 August 2021

Academic Editor: Riaz Ahmad

Copyright $(2021$ Hui Zhang. This is an open access article distributed under the Creative Commons Attribution License, which permits unrestricted use, distribution, and reproduction in any medium, provided the original work is properly cited.

In this paper, we prove the energy conservation for the weak solutions of the $3 \mathrm{D}$ tropical climate model under some sufficient conditions. Our results are similar to Onsager's conjecture which is on energy conservation for weak solutions of Euler equations.

\section{Introduction}

The incompressible Euler equations in $\mathbb{R}^{3}$ are read as follows:

$$
\left\{\begin{array}{l}
\partial_{t} u+u \cdot \nabla u+\nabla p=0 \\
\nabla \cdot u=0 \\
u(x, 0)=u_{0}(x)
\end{array}\right.
$$

where $u$ is the velocity and $p$ is the pressure.

Given that the solution to system (1) is sufficiently smooth, it is easy to see that the total kinetic energy of the flow is conserved. However, turbulent flows are highly irregular; one major character of turbulent fluids is the energy dissipation. In the context of hydrodynamics, this leads to the famous Onsager's conjecture [1]. Specifically, Onsager's conjecture that every weak solution to the Euler equations with Hölder continuity exponent $\alpha>(1 / 3)$ conserves energy; and anomalous dissipation of energy occurs when $\alpha<(1 / 3)$. The first part of the conjecture was proved by Eyink [2] and extended to Besov space in subsequent work of Constantin et al. [3], while significant progress has recently been made on the second part, for example, see $[4,5]$, and some prior work on the compressible Onsager's conjecture can be found in the literature, for example, see $[6,7]$.

The object of this study is to address the relation between the energy conservation and the degree of regularity of the solution for the tropical climate model in $\Omega=[0,1]^{3}$ with periodic boundary conditions

$$
\left\{\begin{array}{l}
\partial_{t} u+u \cdot \nabla u+\nabla p+\nabla \cdot(v \otimes v)=0 \\
\partial_{t} v+v \cdot \nabla u+u \cdot \nabla v+\nabla \theta=0 \\
\partial_{t} \theta+u \cdot \nabla \theta+\nabla \cdot v=0 \\
\nabla \cdot u=0
\end{array}\right.
$$

with initial data

$$
\begin{aligned}
& u(x, 0)=u_{0}(x), \\
& v(x, 0)=v_{0}(x), \\
& \theta(x, 0)=\theta_{0}(x),
\end{aligned}
$$

where $u$ and $v$ denote the vector fields and $\theta$ and $p$ denote the temperature and the pressure, respectively.

The system (2) is derived from the inviscid primitive equations by Frierson et al. [8]. For more details of the related background about the tropical climate model, for instance, see $[9,10]$ and the references therein. Here, it is worth particularly mentioning the solution of (2) with enough regularity to justify integration by parts, and it is clear that the following total energy is conserved:

$$
\text { total energy: } \begin{aligned}
E(t)= & \|u(x, t)\|_{L^{2}(\Omega)}^{2}+\|v(x, t)\|_{L^{2}(\Omega)}^{2} \\
& +\|\theta(x, t)\|_{L^{2}(\Omega)}^{2} .
\end{aligned}
$$


So, it seems very reasonable to investigate the analogue of Onsager's conjecture about system (2). To the best of our knowledge, there is no available result for the weak solutions of the tropical climate model. Our study is motivated by $[3,11]$. However, we are unable to obtain the desired result using the same processes as $[3,12]$. The obstacle is due to $\nabla \cdot v \neq 0$ and the nonlinear term $u \cdot \nabla \theta$. In fact, we require a stronger regularity about $v$ due to the presence of the term $u \nabla \cdot v$. Now, we are ready to state the main results.

Theorem 1. Let $u \in L^{3}\left([0, T] ; B_{3}^{\alpha, \infty}(\Omega)\right) \cap C([0, T]$, $\left.L^{2}(\Omega)\right), \quad \nabla \cdot v \in L^{3}\left([0, T] ; L^{3}(\Omega)\right), \quad v \in L^{3}\left([0, T] ; B_{3}^{\beta, \infty}\right.$ $(\Omega)) \cap C\left([0, T], L^{2}(\Omega)\right)$, and $\theta \in L^{3}\left([0, T] ; B_{3}^{\gamma, \infty}(\Omega)\right) \cap C$ $\left([0, T], L^{2}(\Omega)\right)$ be a weak solution of systems (2) and (3), i.e.,

$$
\begin{aligned}
\left(u, \partial_{t} \varphi\right)+(u \otimes u, \nabla \varphi)+(p, \nabla \varphi)+(v \otimes v, \nabla \varphi) & =0, \\
\left(v, \partial_{t} \varphi\right)+(u \otimes v, \nabla \varphi)+(v \otimes u, \nabla \varphi)+(u \cdot \nabla v, \varphi)+(\theta, \nabla \cdot \varphi) & =0, \\
\left(\theta, \partial_{t} \phi\right)+(u \theta, \nabla \phi)+(v, \nabla \phi) & =0,
\end{aligned}
$$

where $\varphi \in C_{c}^{\infty}\left(\Omega \times R^{+}\right)$is a vector field and $\phi \in C_{c}^{\infty}\left(\Omega \times R^{+}\right)$ is a scalar field. If

$$
\begin{array}{r}
\alpha>\frac{1}{3}, \\
\alpha+2 \beta>1, \\
\alpha+2 \gamma>1,
\end{array}
$$

then we have

$$
E(t)=E(0), \quad \text { for } t \in[0, T] .
$$

\section{Preliminary Remarks}

In this section, we briefly discuss some properties of the Besov space $B_{3}^{\alpha, \infty}(\Omega)$.

$$
\|u\|_{B_{3}^{\alpha, \infty}(\Omega)}:=\|u\|_{L^{3}(\Omega)}+\sup _{x \in \Omega} \frac{\|u(x+y)-u(x)\|_{L^{3}(\Omega)}}{|y|^{\alpha}}<+\infty .
$$

From the above definition, we have the following inequalities [3]:

$$
\|u(x+y)-u(x)\|_{L^{3}(\Omega)} \leq C|y|^{\alpha}\|u\|_{B_{3}^{\alpha, \infty}(\Omega)} .
$$

Let $\eta \in C_{c}^{\infty}\left(\mathbb{R}^{3}\right)$ be a standard mollifying kernel and set

$$
\eta^{\epsilon}=\frac{1}{\epsilon^{3}} \eta\left(\frac{x}{\epsilon}\right) \text {. }
$$

With the notation

$$
\begin{aligned}
u^{\epsilon} & =\eta^{\epsilon} * u, \\
x \in \Omega^{\epsilon} & =\{x \in \Omega: \operatorname{dist}(x, \partial \Omega)>\epsilon\},
\end{aligned}
$$

we have the following inequalities [3]:

$\left\|u-u^{\epsilon}\right\|_{L^{3}} \leq C \epsilon^{\alpha}\|u\|_{B_{3}^{\alpha, \infty}(\Omega)}$,

$$
\begin{aligned}
\left\|\nabla u^{\epsilon}\right\|_{L^{3}} & \leq C \epsilon^{\alpha-1}\|u\|_{B_{3}^{\alpha, \infty}(\Omega)}, \\
\left\|u^{\epsilon}\right\|_{L^{3}} & \leq\left\|u-u^{\epsilon}\right\|_{L^{3}}+\|u\|_{L^{3}} \leq\left(C \epsilon^{\alpha}+1\right)\|u\|_{B_{3}^{\alpha, \infty}(\Omega)} .
\end{aligned}
$$

Now, we define

$$
\begin{aligned}
& r_{\epsilon}(u, v)(x)=\int_{\Omega} \eta^{\epsilon}(y)[(u(x-y)-u(x)) \otimes(v(x-y)-v(x))] \mathrm{d} y \\
& q_{\epsilon}(u, v)(x)=\int_{\Omega} \eta^{\epsilon}(y)[(u(x-y)-u(x)) \nabla \cdot(v(x-y)-v(x))] \mathrm{d} y \\
& h_{\epsilon}(u, \theta)(x)=\int_{\Omega} \eta^{\epsilon}(y)[(u(x-y)-u(x))(\theta(x-y)-\theta(x))] \mathrm{d} y
\end{aligned}
$$

Then, it is easy to check the following equalities, it is pointed out that the first equality was discovered in [3]:

$$
\begin{aligned}
(u \otimes v)^{\epsilon} & =u^{\epsilon} \otimes v^{\epsilon}+r_{\epsilon}(u, v)-\left(u-u^{\epsilon}\right) \otimes\left(v-v^{\epsilon}\right), \\
(u \nabla \cdot v)^{\epsilon} & =u^{\epsilon} \nabla \cdot v^{\epsilon}+q_{\epsilon}(u, v)-\left(u-u^{\epsilon}\right) \nabla \cdot\left(v-v^{\epsilon}\right), \\
(u \theta)^{\epsilon} & =u^{\epsilon} \theta^{\epsilon}+h_{\epsilon}(u, \theta)-\left(u-u^{\epsilon}\right)\left(\theta-\theta^{\epsilon}\right) .
\end{aligned}
$$

\section{Proof of the Main Theorem}

For the sake of simplicity, we will proceed as if the solution is differentiable in time. The extra arguments need to be modifed in time are straightforward.
Proof. First, we apply the test function $\varphi=\left(u^{\epsilon}\right)^{\epsilon}$ on the first equation and then we have

$$
\begin{aligned}
\frac{1}{2} \frac{\mathrm{d}}{\mathrm{d} t} \int_{\Omega}\left|u^{\epsilon}(x, t)\right|^{2} \mathrm{~d} x & =\int_{\Omega}(u \otimes u)^{\epsilon} \nabla u^{\epsilon} \mathrm{d} x+\int_{\Omega}(v \otimes v)^{\epsilon} \nabla u^{\epsilon} \mathrm{d} x \\
& =I_{1}+I_{2} .
\end{aligned}
$$

We point out that the $I_{1}$ term can be estimated as same as that in [3], and we omit the details:

$$
\left|I_{1}\right| \leq C \epsilon^{3 \alpha-1}\|u\|_{B_{3}^{\alpha, \infty}(\Omega)}^{3} .
$$

Now, we estimate $I_{2}$ : 


$$
\begin{aligned}
I_{2} & =\int_{\Omega}(v \otimes v)^{\epsilon} \nabla u^{\epsilon} \mathrm{d} x \\
& =\int_{\Omega}\left[v^{\epsilon} \otimes v^{\epsilon}+r_{\epsilon}(v, v)-\left(v-v^{\epsilon}\right) \otimes\left(v-v^{\epsilon}\right)\right] \nabla u^{\epsilon} \mathrm{d} x \mathrm{~d} t \\
& =I_{21}+I_{22}+I_{23} .
\end{aligned}
$$

Using the following inequality and (12) and (13), we obtain

$$
\left\|r_{\epsilon}(v, v)\right\|_{L^{(3 / 2)}} \leq C \epsilon^{2 \beta}\|v\|_{B_{3}^{\beta, \infty}(\Omega)}^{2} .
$$

We can also obtain the following estimate through the above inequalities:

$$
\left|I_{22}\right| ;\left|I_{23}\right| \leq C \epsilon^{\alpha+2 \beta-1}\|v\|_{B_{3}^{\beta, \infty}(\Omega)}^{2}\|u\|_{B_{3}^{\alpha, \infty}(\Omega)} .
$$

Because $\nabla \cdot v \neq 0, I_{21}$ cannot be zero, and we will estimate this term later.

We rewrite the second equation as follows:

$$
\partial_{t} v+\nabla \cdot(u \otimes v)+\nabla \cdot(v \otimes u)-u \nabla \cdot v+\nabla \theta=0 .
$$

Second, we apply the test function $\varphi=\left(v^{\epsilon}\right)^{\epsilon}$ on the above equation and then we have

$$
\begin{aligned}
\frac{1}{2} \frac{\mathrm{d}}{\mathrm{d} t}\left\|v^{\epsilon}\right\|_{2}^{2}= & \left.\int_{\Omega}(u \otimes v)^{\epsilon} \nabla v^{\epsilon} \mathrm{d} x+\int_{\Omega}(v \otimes u)^{\epsilon}\right] \nabla v^{\epsilon} \mathrm{d} x \\
& +\int_{\Omega}(u \nabla \cdot v)^{\epsilon} v^{\epsilon} \mathrm{d} x+\int_{\Omega} \theta^{\epsilon} \nabla \cdot v^{\epsilon} \mathrm{d} x \\
= & H_{1}+H_{2}+H_{3}+H_{4} .
\end{aligned}
$$

Using (12), one can easily obtain

$$
\left\|r_{\epsilon}(u, v)\right\|_{L^{(3 / 2)}} \leq C \epsilon^{\alpha+\beta}\|u\|_{B_{3}^{\alpha, \infty}(\Omega)}\|v\|_{B_{3}^{\beta, \infty}(\Omega)} .
$$

Then,

$$
\begin{aligned}
H_{1} & =\int_{\Omega}\left[u^{\epsilon} \otimes v^{\epsilon}+r_{\epsilon}(u, v)-\left(u-u^{\epsilon}\right) \otimes\left(v-v^{\epsilon}\right)\right] \nabla v^{\epsilon} \mathrm{d} x \\
& =H_{11}+H_{12}+H_{13} .
\end{aligned}
$$

Similar to (21), we have

$$
\left|H_{12}\right| ;\left|H_{13}\right| \leq C \epsilon^{\alpha+2 \beta-1}\|v\|_{B_{3}^{\beta, \infty}(\Omega)}^{2}\|u\|_{B_{3}^{\alpha, \infty}(\Omega)} .
$$

We can rewrite $H_{11}$ as follows:

$$
\begin{aligned}
H_{11} & =-\int_{\Omega} \nabla \cdot\left(u^{\epsilon} \otimes v^{\epsilon}\right) v^{\epsilon} \mathrm{d} x \\
& =-\int_{\Omega} v^{\epsilon} \cdot \nabla u^{\epsilon} v^{\epsilon} \mathrm{d} x-\int_{\Omega} u^{\epsilon} v^{\epsilon} \nabla \cdot v^{\epsilon} \mathrm{d} x \\
& =-\int_{\Omega}\left(v^{\epsilon} \otimes v^{\epsilon}\right) \nabla u^{\epsilon} \mathrm{d} x-\int_{\Omega} u^{\epsilon} v^{\epsilon} \nabla \cdot v^{\epsilon} \mathrm{d} x .
\end{aligned}
$$

We can estimate $\mathrm{H}_{2}$ using a similar process as follows:

$$
\begin{aligned}
H_{2} & =\int_{\Omega}\left[v^{\epsilon} \otimes u^{\epsilon}+r_{\epsilon}(v, u)-\left(v-v^{\epsilon}\right) \otimes\left(u-u^{\epsilon}\right)\right] \nabla v^{\epsilon} \mathrm{d} x \\
& =H_{21}+H_{22}+H_{23} .
\end{aligned}
$$

Then, we have the following estimate:

$$
\left|H_{22}\right| ;\left|H_{23}\right| \leq C \epsilon^{\alpha+2 \beta-1}\|v\|_{B_{3}^{\beta, \infty}(\Omega)}^{2}\|u\|_{B_{3}^{\alpha, \infty}(\Omega)} .
$$

Here, we have used the following equality due to $\nabla \cdot u=0$ :

$$
\begin{aligned}
H_{21} & =-\int_{\Omega} \nabla \cdot\left(v^{\epsilon} \otimes u^{\epsilon}\right) v^{\epsilon} \mathrm{d} x \\
& =-\int_{\Omega} u^{\epsilon} \cdot \nabla v^{\epsilon} v^{\epsilon} \mathrm{d} x-\int_{\Omega} u^{\epsilon} v^{\epsilon} \nabla \cdot u^{\epsilon} \mathrm{d} x \\
& =0 .
\end{aligned}
$$

Now, we estimate $\mathrm{H}_{3}$ as follows:

$$
\begin{aligned}
H_{3} & =\int_{\Omega}\left[u^{\epsilon} \nabla \cdot v^{\epsilon}+q_{\epsilon}(u, v)-\left(u-u^{\epsilon}\right) \nabla \cdot\left(v-v^{\epsilon}\right)\right] v^{\epsilon} \mathrm{d} x \\
& =H_{31}+H_{32}+H_{33} .
\end{aligned}
$$

Using (12), one can obtain the following inequality:

$$
\left\|q_{\epsilon}(u, v)\right\|_{L^{(3 / 2)}} \leq C \epsilon^{\alpha}\|u\|_{B_{3}^{\alpha, \infty}(\Omega)}\|\nabla \cdot v\|_{L^{3}(\Omega)} .
$$

Then, we have

$$
\left|H_{32}\right| ;\left|H_{33}\right| \leq C \epsilon^{\alpha}\left(C \epsilon^{\beta}+1\right)\|\nabla \cdot v\|_{L^{3}(\Omega)}\|u\|_{B_{3}^{\alpha, \infty}(\Omega)}\|v\|_{B_{3}^{\beta, \infty}(\Omega)} \text {. }
$$

From the above, we can easily find that

$$
I_{21}+H_{11}+H_{31}=0 \text {. }
$$

We will estimate $H_{4}$ later. At last, we apply the test function $\phi=\left(\theta^{\epsilon}\right)^{\epsilon}$ on the first equation and then we have $\frac{1}{2} \frac{\mathrm{d}}{\mathrm{d} t}\left\|\theta^{\epsilon}\right\|_{L^{2}(\Omega)}^{2}=\int_{\Omega}(u \theta)^{\epsilon} \nabla \theta^{\epsilon} \mathrm{d} x-\int_{\Omega} \nabla \cdot v^{\epsilon} \theta^{\epsilon} \mathrm{d} x=N_{1}+N_{2}$.

Now, we have

$$
\begin{aligned}
N_{1} & =\int_{\Omega}\left[u^{\epsilon} \theta^{\epsilon}+h_{\epsilon}(u, \theta)-\left(u-u^{\epsilon}\right)\left(\theta-\theta^{\epsilon}\right)\right] \nabla \theta^{\epsilon} \mathrm{d} x \\
& =N_{11}+N_{12}+N_{13} .
\end{aligned}
$$

Similarly, we also have the following inequality:

$$
\left\|h_{\epsilon}(u, \theta)\right\|_{L^{(3 / 2)}} \leq C \epsilon^{\alpha+\gamma}\|u\|_{B_{3}^{\alpha, \infty}(\Omega)}\|\theta\|_{B_{3}^{\gamma, \infty}(\Omega)} .
$$

Then, we have

$$
\left|N_{2}\right| ;\left|N_{3}\right| \leq C \epsilon^{\alpha+2 \gamma-1}\|u\|_{B_{3}^{\alpha, \infty}(\Omega)}\|\theta\|_{B_{3}^{\gamma, \infty}(\Omega)}^{2} .
$$

Because $\nabla \cdot u^{\epsilon}=0$, we can easily find that 


$$
N_{11}=0 .
$$

We can also see that

$$
H_{4}+N_{2}=0 \text {. }
$$

Finally, we can establish the following inequality through the above estimates and condition (6):

$$
\left|E^{\epsilon}(t)-E^{\epsilon}(0)\right| \leq C \epsilon^{3 \alpha-1}+C \epsilon^{\alpha+2 \beta-1}+C \epsilon^{\alpha+2 \gamma-1} \longrightarrow 0,
$$

as $\epsilon \longrightarrow 0$, which completes the proof.

\section{Data Availability}

The data used to support the findings of this study are included within the article.

\section{Conflicts of Interest}

The author declares that there are no conflicts of interest.

\section{Acknowledgments}

Hui Zhang was partially supported by the NSFC (no. 31971185) and Anhui Education Bureau (no. KJ2019A0556).

\section{References}

[1] L. Onsager, "Statistical hydrodynamics," Il Nuovo Cimento, vol. 6, no. S2, pp. 279-287, 1949.

[2] G. L. Eyink, "Energy dissipation without viscosity in ideal hydrodynamics I. Fourier analysis and local energy transfer," Physica D: Nonlinear Phenomena, vol. 78, no. 3-4, pp. 222240, 1994.

[3] P. Constantin, E. Weinan, and E. S. Titi, "Onsager's conjecture on the energy conservation for solutions of Euler's equation," Communications in Mathematical Physics, vol. 165, no. 1, pp. 207-209, 1994.

[4] C. De Lellis and L. Székelyhidi Jr., "Dissipative continuous Euler flows," Inventiones Mathematicae, vol. 193, no. 2, pp. 377-407, 2013.

[5] C. De Lellis and L. Székelyhidi Jr., "Dissipative Euler flows and Onsager's conjecture," Journal of the European Mathematical Society, vol. 16, no. 7, pp. 1467-1505, 2014.

[6] T. D. Drivas and G. L. Eyink, "An onsager singularity theorem for turbulent solutions of compressible Euler equations," Communications in Mathematical Physics, vol. 359, no. 2, pp. 733-763, 2018.

[7] E. Feireisl, P. Gwiazda, A. Świerczewska-Gwiazda, and E. Wiedemann, "Regularity and energy conservation for the compressible Euler equations," Archive for Rational Mechanics and Analysis, vol. 223, no. 3, pp. 1375-1395, 2017.

[8] D. M. W. Frierson, A. J. Majda, and O. M. Pauluis, "Large scale dynamics of precipitation fronts in the tropical atmosphere: a novel relaxation limit," Communications in Mathematical Sciences, vol. 2, no. 4, pp. 591-626, 2004.

[9] A. J. Majda and J. A. Biello, "The nonlinear interaction of barotropic and equatorial baroclinic Rossby waves," Journal of the Atmospheric Sciences, vol. 60, no. 15, pp. 1809-1821, 2003.

[10] T. Matsuno, "Quasi-geostrophic motions in the equatorial area," Journal of the Meteorological Society of Japan, vol. 44, pp. 25-42, 1996.
[11] H. Zhang, "Energy conservation for the 3D tropical climate model in bounded domains," Journal of Mathematical Analysis and Applications, vol. 492, no. 1, Article ID 124424, 2020.

[12] R. E. Caflishch, I. Klapper, and G. Steele, "Remarks on singularities, dimension and energy dissipation for idea hydrodynamics and MHD," Communications in Mathematical Physics, vol. 184, pp. 443-455, 1997. 